\title{
Theoretical Spectrum of Mass of the Nucleons: New Aspects of the QM
}

\author{
Giovanni Guido (10) \\ Department of Physics and Mathematics, High School “C. Cavalleri” Parabiago, Milano, Italy \\ Email: gioguido54@gmail.com
}

How to cite this paper: Guido, G. (2021) Theoretical Spectrum of Mass of the Nucleons: New Aspects of the QM. Journal of High Energy Physics, Gravitation and Cosmology, 7, 123-143. https://doi.org/10.4236/jhepgc.2021.71006

Received: November 3, 2020

Accepted: January 5, 2021

Published: January 8, 2021

Copyright (c) 2021 by author(s) and Scientific Research Publishing Inc. This work is licensed under the Creative Commons Attribution International License (CC BY 4.0).

http://creativecommons.org/licenses/by/4.0/

\begin{abstract}
In this paper, we show a new theoretical procedure for calculating the nucleonic mass values. We develop this procedure on the geometric representation of $(\mathrm{u}$, d) quarks, these seen as golden structures of coupled quantum oscillators (Aureum Geometric Model or AGM). Using AGM, we also build the geometric structures of nucleons $(\mathrm{p}, \mathrm{n})$, determining their structure equations and spins. Thank AGM, coherent to QCD, new aspects of the Quantum Mechanics emerge, opening to anew descriptive paradigm in Particle Physics.
\end{abstract}

\section{Keywords}

Quark, Structure Equation, Geometric Structure, Golden Number, Interpenetration, Iquo, Gluon, Proton, Neutron

\section{Introduction}

One of the more problematic aspects in Hadronic literature [1] [2] is that one in which only a small fraction of the hadron mass seems to be associated with the bare mass of the elementary quarks. If the total bare mass of quarks $(u, u, d)$ forming the proton (for literature) is around $10 \mathrm{MeV}$ [3], then quarks represent only $\sim 1 \%$ of the proton mass. The same occurs in a pion or, generally, in mesons. Nowadays, in literature [4] [5], one of the answers to this mystery of mass origin in hadrons is that the remaining mass fraction could be due to the force (gluons) binding the quarks within hadrons, i.e., Quantum Chromo Dynamics (QCD). The researchers [4] [5], by particular discretization of potentials formulated in QCD, have found the values of the "bare" masses of quarks corresponding, obtained directly in lattice simulations [6]. Furthermore, these values and others of experimental masses [5] are used as input (see the pion mass) to improve the mass values and coupling parameters in the interactions and so to 
calculate the masses of hadrons (see later in the conclusions of this paper). Nevertheless, the procedures of calculation are very complicated, also if supported by processed programs of calculation with computers. Besides, lattice QCD is an expensive technique, because the discretization creates errors, and to remove them entails taking the lattice spacing, $a$, to zero. In a set of studies, for remedying to these complex problems of calculation and calculating with simply way the mass values of light mesons, we have used another path [7] [8] [9]. Instead of incorporate the binding energy of the quarks into appropriate potentials of the binding quanta (gluons), see the QCD, we incorporated this energy into the mass of quarks which compose the pion [7]. We have so obtained the values of "dressed" mass of the ( $u, d)$ quarks, derived, nevertheless, from a suitable "geometric structure" of the pions [7] [8] and same quarks [9]. These mass values are very greater than that of free quarks. This way, the bonded quarks inside the hadron would have enough mass to reach the hadron mass value they compose, provided that we correctly sum them up. Since the pion, the lightest meson, is made of two bonded states of quarks, its mass could be placed as base mass to determine the mass of the most massive mesons and hadrons (as it happens in literature [10] [11]. We already proved in two separate articles [8] [9] that all light mesons, following the pion, are elaborated structures of pions lattice $\left\{\pi^{+}\right.$, $\left.\pi^{-}\right\}$and $\{d, \underline{d}\}$-lattice of $\mathrm{d}$-quarks. This hypothesis induces us to admit an appropriate structure equation [7] [8] [9], which describes all mesons and thus also hadrons. By this equation, we can so calculate the masses of light mesons and hadrons. Moreover, the structure equation allows us to identify the possible decays of a particle and its spin value. Nevertheless, all of this (the calculation procedure) is possible only if we affirm [12] [13] [7] the quarks having a well-defined geometric structure. In this way, we cannot consider a particle (quark) anymore as an object-point, see the Quantum Relativistic Theory of fields and QCD [14] [15]). One can achieve the idea of a geometric structure of quarks if we assume a quark made by a non-separable set of coupled quantum oscillators with "Aurea" (golden) geometric structure [12] [7]. We have referred to this as the Aureum Geometric Model (AGM) of quarks. Thanks to the quarks geometric hypothesis, it is possible to explain fundamental issues such as the origin of the mass of the hadrons, the role of gluons in constructing bonded quarks and the existence of molecules of quarks and also pions [8] [9]. We can achieve all of this without turning to the QCD and its calculations by colour potentials. Nevertheless, for obtaining the hadronic masses, we need to revise the mass conception in physics and to introduce a new idea of mass calculation ( $\otimes$-operation) [7]. The idea of calculation takes into account both interactions between quarks and the possible interpenetration [8] [9]. This last aspect is purely quantum-undulatory like the superposition of particles-waves. The latter may exist if we admit that two different structures (states) of coupled quantum oscillators (or quarks) can overlap without exchanging energy. The global mass of hadron must so take in account all the possible configurations of quark components, both the ones with inter- 
penetration and the ones with interactions. In this way, the hypothesis of the quark geometrical structure introduces a "new paradigm" [8] in the phenomenology of hadronic interactions. Thanks to a few primary hypotheses, the new paradigm allows describing the hadronic phenomenology in a more structured and straight forward way of that of QCD. The first evidence is the spectrum of light mesons with mass values very closed (if not even equal) to the experimental ones that we have calculated in two papers, see ref. [8] [9]. In this paper, exactly, we will calculate, by theoretical physics aspects and suitable mathematical procedure, the masses of the nucleons, while in next study that of light baryons without strangeness $\left(\Delta^{\circ}, \Delta^{-}, \Delta^{++}\right)$. In finally, in the conclusions section of this paper, we will show that the two paths, AGM and QCD, are physical equivalents.

\section{The Geometric Structure of Particles}

\subsection{The Dressed Masses and Bare of Quarks $(u, d)$}

In ref. [12], we showed that each quark $(u, d)$ of the proton has an "aurea" geometric structure (golden structure) achieved by coupling of three quantum oscillators (vertices of a triangle), called IQuO, the acronym of Intrinsic Quantum Oscillator. This coupling consists of the IQuO quantum oscillators of the junction (junction gluons), placed along the sides. We showed that three quantum oscillators, in eigenstate $n=2$, and connected by junction quantum oscillators (with $n=1, n=2$ ), can couple for origin an "aurea" triangular structure. Thanks to this conjecture, we have also given a geometric structure to mesons [9]: coupling among two golden triangles $(u, d)$ which are bounded by gluons (binding gluons), see Figure 1.

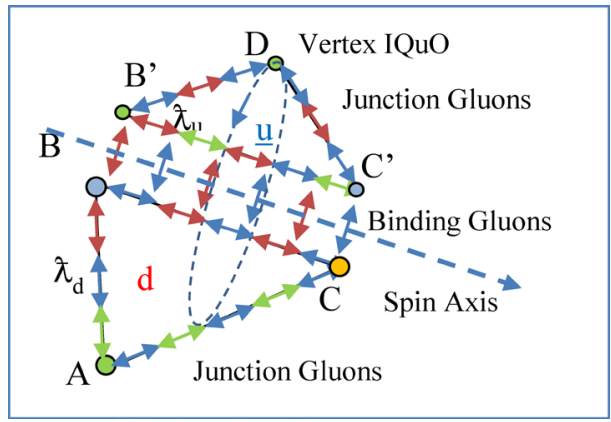

Figure 1. Meson with gluons.

By the "aurea" hypothesis, we have calculated the mass values of quarks inside the pion $\left(u_{\pi}, d_{\pi}\right)$ by equations' system:

$$
\left\{\begin{array}{l}
\underline{m}\left(u_{\pi}\right)+\underline{m}\left(d_{\pi}\right)=m_{\pi}^{ \pm} \\
\underline{m}\left(d_{\pi}\right) / \underline{m}\left(u_{\pi}\right)=\phi
\end{array}\right.
$$

where is $\phi$ the golden number. This equation has solutions $\left[m\left(u_{\pi}\right)=(53.31)\right.$ $\mathrm{MeV}, m\left(d_{\pi}\right)=(86.26) \mathrm{MeV}$ ] with $m\left(\pi^{ \pm}\right)=(139.57) \mathrm{MeV}$. We assign these mass values to the physical system (pion) composed by quarks $\left(u_{\pi} d_{\pi}\right)$ and binding 
gluons: we speak of "dressed" quarks. Any observer detects the couple $\left(u_{\pi}, d_{\pi}\right)$ as a unique quantum system (pion $\pi$ ) with non-separated components: we cannot detect an only oscillator of the structure. Besides, we obtained the mass values of bare quarks considering the mass defect between the charged pion and the neutral one, $\Delta m\left(\pi^{ \pm}, \pi^{0}\right)$, by equations' system, see ref. [8]:

$$
\left\{\begin{array} { l } 
{ ( 1 / 2 ) [ m ( u _ { f } ) + m ( d _ { f } ) ] = \Delta m _ { \pi } ^ { 0 } } \\
{ m ( d _ { f } ) / m ( u _ { f } ) = \phi }
\end{array} \Rightarrow \left\{\begin{array}{l}
m\left(u_{f}\right)=(3.51) \mathrm{MeV} \\
m\left(d_{f}\right)=(5.67) \mathrm{MeV}
\end{array}\right.\right.
$$

\subsection{The Proton Structure}

Also, the proton can be described by a structure equation relative to an "aurea" geometric structure: three golden triangles couple building a geometric figure which can change taking the form of a pentagon or triangle, see Figure 2. This idea [7] of aurea structure is born by the observation that between the Compton's wave lengths $\left(\Delta_{p l}\right)$ of the Planck-particle and the one of the proton $\left(\Delta_{p}\right)$ there is an aurea (golden) relation $\left[\left(\Delta_{p} / \Delta_{p l}\right) \propto \phi\right]$, see Figure 2 .

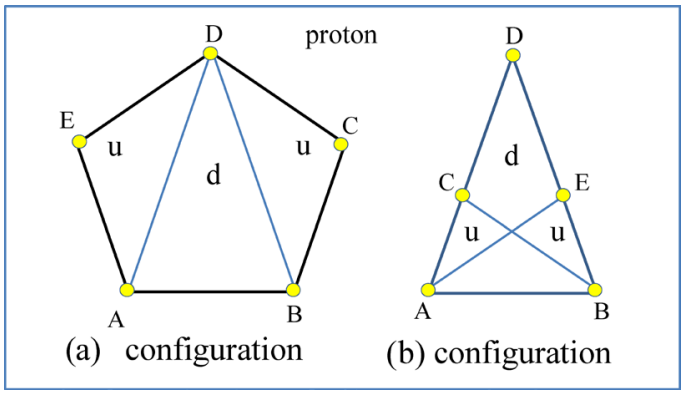

Figure 2. Two configurations of the proton in two different times.

Where $(\phi)$ is the "aureus" (golden) number.

The configurations (a) and (b) belong in different times to the same particle because the u-quarks are in rotation around the sides (AD), (DB). Note the three vertices-IQuO can constitute the three scattering centers seen in the experiments of deep inelastic diffusion (e, p). In the first time, the geometric representation of quarks (AGM) can be seen differently to those of partons and QCD. In AGM, the quark is not a point-particle surrounded by clouds of gluons but a set of coupled oscillators (IQuO) by of junction oscillators ("junction gluons"). In its turn, each quark can be bound to other quarks by "binding gluons". Nevertheless, studying more in-depth these new aspects, we can see the two models (QCD and AGM) having some points of connection. In fact, in QCD [14] [16] the quarks ( $u$, d) would show, in the periphery, an interaction potential $[V(r) \approx k r]$, with $r$ the linear nucleon dimensions, expressed by an interaction strong force with modality $[F \approx k]$. This force should be similar at the interaction force in AGM. In this theory, the binding gluons in the geometric structure of two quarks $(u, d)$ are equivalent to simple oscillators with elastic force $[F \approx \underline{k} x]$. Specifically, $\underline{k}$ is a variable elastic parameter appropriated: in the periphery the force is constant $[F \approx k]$, 
see the Cornel potential in ref. [14]. These correlations so point out two similar paths for treating the Hadrons, even if the AGM admits the innovation of a geometric structure of quarks. This assert introduces a "new paradigm" [8] [9] in the representation of particles and phenomenology of their hadronic interactions. To the geometric structure of quarks $(u, d)$, see later, we associate a structure equation expressed [7] by matrices [ $\|u\|,\|d\|]$ : the terms of each matrix are quantum vertex-oscillators of single quark, expressed by the quantum operators $\left(a, a^{+}\right)$, in the IQuO form. Tanks to the structure equation, we have calculated the mass of light mesons [9]. In this study, we will show that by structure equation one can determine the spectrum of the mass value of elementary baryons as the nucleons.

\subsection{The Quark's Interpenetration}

The structures are not rigid because the binding gluons allow the movement of quarks: the quarks reciprocally rotate one respect to other around to a common axis. As noted [7] [9], different relative orientations between $u$ quarks and $d$ quarks imply a relative rotation (orbital spin), suggesting a mutual crossing of the quarks (see Figure 1). This crossing can have the meaning of a reciprocal interpenetration $(\otimes)$ between quarks $\left(q_{i} \otimes q_{j}\right)$, see the pion in ref. [7]. In this way, we can consider the pion as a structure of two quarks but seen as a uniqueness: $\left[\pi^{+}=(u \underline{\otimes})\right]$. The $\underline{\otimes}$ operation is a combination of two operations $(\oplus, \otimes)$, where the $(\oplus)$ is the representative operation of dynamics interaction, while the $(\otimes)$ is the operation of interpenetration. During this mutual crossing, quarks no energy exchange between them in those parts that interpenetrate (in the diagonal BC of Figure 1, instead there is an exchange of binding quanta, see gluons). An analogous phenomenon occurs in crossing waves. The idea of interpenetration must be considered as mere "representation" of a quantum state associated with different configurations, see Figure 2: recall spin the idea associated with different possibilities of spatial orientations. Thus, interpenetration and spin are reciprocally correlated [Interpenetration $\Leftrightarrow$ Spin] in a quantum way. The interpenetration idea leads us also to review the concept of mass of a composite structured (particle), where we need to consider all possible configurations of its structural components. Besides, note that the interpenetration between quarks implies a reciprocal "intrinsic internal movement". We can associate at this movement a kinetic energy form, called interpenetration kinetic energy [9]. We think that interpenetration kinetic energy of all possible configurations involves both the global mass value (matrix of mass defects) and the spin: in fact, recall the spin is important in interactions between particles.

\subsection{The Correlation between Quark's Interpenetration and Spin}

The different configurations, or orientations, can induce us to think about the orbital spin $\left(s_{l}\right)$ of each quark. To individual quark, seen like Fermion particle, one associates an intrinsic spin of quarks $\left(s_{q}\right)$, while now we associate also the gluons' spin $\left(s_{g}\right)$ to rotations of u-quark (or d-quark) around the $\mathrm{X}$-axis (see the 
experimental observations about proton spin [17]). The hypothesis of structure does not conflict with experiments [18] of CERN (COMPASS), SLAC, and DESY (HERMES), where the spin of the proton is experimentally the sum of intrinsic spins $\left(s_{q}\right)$ of quarks with their orbital motions $\left(s_{I}\right)$ and their gluons $\left(s_{g}\right)$. In this way, in AGM the total spin of the proton is the sum of different components of spin: $\left[s_{\pi}=s_{q}+s_{l}+s_{g}\right]$. Just in ref. [18] experiments conducted at the CERN, DESY, JLab, RHIC, and SLAC laboratories have revealed that the spin of its quark constituents carries only about $30 \%$ of the proton's spin. Then, we will have:

$$
s_{p}=\left\{\begin{array}{l}
s_{\text {tot }}(q)=\left[(\uparrow \downarrow)_{q_{1}},(\uparrow \downarrow)_{q_{2}},(\uparrow \downarrow)_{q_{3}}\right] \\
s_{\text {tot }}(l)=\left[(\uparrow \downarrow)_{q_{1}},(\uparrow \downarrow)_{q_{2}},(\uparrow \downarrow)_{q_{3}}\right] \\
s_{\text {tot }}(g)=\left[(\uparrow \downarrow)_{q_{1}},(\uparrow \downarrow)_{q_{2}},(\uparrow \downarrow)_{q_{3}}\right]
\end{array}\right\}=\left\{s_{\text {tot }}(q)+s_{\text {tot }}(l)+s_{\text {tot }}(g)\right\}
$$

where [ $\left.\left(s_{\text {tot }}(q)= \pm 1 / 2, \pm 3 / 2\right), s_{\text {tot }}(I)= \pm 1, s_{t o t}(g)=\mp 1\right]$. In AGM, the structure of three coupled IQuO needs that $\left[s_{l}=-s_{g}\right]$ : in this way, the proton spin is $\left[s_{t o t}(p)=\right.$ $\left.s_{\text {tot }}(q)= \pm 1 / 2\right]$.

\subsection{The $(\otimes, \oplus)$ Operations and the $\left(F_{m} F_{m}\right)$ Function}

In the interpenetration of quarks and their dynamics interactions, we used, see ref. [7] [9], a new $\underline{\otimes}$-operation of combination (or coupling) of quarks and particles: $\left(a_{i} \otimes b_{j}\right)$. The new operation $(\underline{\otimes})$ indicates a composition of two operations $(\otimes, \oplus)$ or $[\underline{\otimes} \equiv(\otimes U \Theta)]$, where $\otimes$-operation describes the proper interpenetration of the quarks and follows, in algebraic calculations, the properties of multiplication. Instead, $\oplus$-operation describes dynamics interactions and follows, in algebraic calculations, the properties of the sum. In ref. [9], see Table 1 and Table 2 , we listed some algebraic properties of the $\underline{\otimes}$-operation, used in the calculations of mesons' masses. These properties now we will use in this paper in calculations of hadrons' masses (nucleons).For the calculation of meson masses and mass defects, we have used two functions $\left[\left(F_{m}\right),\left(F_{\Delta m}\right)\right]$. In general, for every $X$-system (composed by more particles), we used [7] [8] [9] the partial mass Function $\left(F_{m}\right)$ applied to structure equation of $X$-system, with

$X\left[\left(A_{1}, A_{2}, \cdots, A_{n}\right)_{\otimes} ;\left(B_{1}, B_{2}, \cdots, B_{n}\right)_{\oplus}\right]$, where $\left[\left(A_{i}\right)_{\otimes},\left(B_{j}\right)_{\oplus}\right]$ are the "base components" of the structure which are combinations of coupling between base particles by of the operations $(\otimes, \oplus)$. The $\left(F_{m}\right)$ is such an application on the structure components $(A, B)$, which gives us the mass values $\left(m_{i}\right)$ of these components of base. The $\left(F_{m}\right)$ operates on $X$, in the following way:

$$
\begin{aligned}
F_{m}(X) & =\left\{\sum_{(i, j)=1}^{n} F_{m}\left[\left(A_{i}\right)_{(\otimes)},\left(B_{j}\right)_{(\oplus)}\right]\right\} \\
& =\left[\sum_{i=1}^{n} m\left(A_{i}\right)_{(\otimes)}\right]_{A}+\left[\sum_{j=1}^{m} m\left(B_{j}\right)_{(\oplus)}\right]_{B} \\
& =\left[m(a \otimes b)_{A_{1}}+\cdots+m(w \otimes z)_{A_{n}}\right]_{A}+\left[m(a \oplus b)_{B_{1}}+\cdots+m(w \oplus z)_{B_{m}}\right]_{B}
\end{aligned}
$$


where $(a, b, \ldots, w, z)$ are the base particles which build the structure (hadron). We will have the following applications:

$$
\left\{\begin{array}{c}
F_{m}\left(A_{(\otimes)}\right)=F_{m}[(a) \otimes(b)]_{A}=\langle m(a, b)\rangle=\langle m(a), m(b)\rangle \\
F_{m}\left(B_{(\oplus)}\right)=F_{m}[(a) \oplus(b)]_{B}=m((a) \oplus(b))=m(a)+m(b)
\end{array}\right\}
$$

Note that the mass of two interpenetrating particles $[a \otimes b]$ is obtained by the average value of individual masses $[<m(a, b)>]$, while the mass of two interacting particles $[a \oplus b]$ is obtained by the sum of the masses of each particle. To obtain the total mass of a structure, it needs to add eventual $\left(m_{k i n}\right)$ relativistic kinetic mass and mass defects $(\Delta m)$. Nevertheless, we can consider the relativistic kinetic mass as a positive defect of mass: $\left(\Delta m_{k i n}\right)>0$.

We will have: $\left.\left[m_{\text {tot }}=m_{\text {part }}+\Delta m_{i}\right)\right]$. Where $(\Delta m)>0,(\Delta m)<0$.

The mass defect will be: $\Delta m=\Delta m_{g}+\Delta m_{e m}$

Where $\Delta m_{g}$ is the mass defect due to binding by gluons. Nevertheless, the $\Delta m_{g}$ has been included in masses of the charged pion (see ref. [7] [9]) or in quark ( $u_{\pi}$ $\left.d_{\pi}\right)$ ); therefore, we consider only electromagnetic mass defect: $\Delta m=\Delta m_{e m}$.

To obtain the mass defects $(\Delta m>0, \Delta m<0)$ we have used [9] a Function $\left(F_{\Delta m}\right)$ applied to structure equation for calculating the mass defects. Besides, it needs to consider that:

$$
\begin{aligned}
& \Delta m[(a) \otimes(b)]_{A_{i}}=\left\{\begin{array}{c}
0 \\
\Delta m(a, b)_{(a \cap b)} \neq 0
\end{array}\right\} \\
& \Delta m[(a) \oplus(b)]_{B_{i}}=\Delta m(a, b)_{\text {interaction }}
\end{aligned}
$$

where the $(a, b)$ point out "base particles" of the $\left(A_{i}, B_{j}\right)$-component, as, i.e. the pions $\pi$ or the quarks $q$. Note mass defect is zero if there is the only interpenetration between the two particles $(a, b)$ without interacting parts. Instead, the mass defect cannot be zero if there are some parts of $(a, b)$ dynamically interacting $(a \oplus b)$, see the neutral pion in diagonal in ref. [9], where the d-quark and u-quark, exchange energy quanta along the diagonal. In ref. [7] we have calculated the various mass defects in dynamics couplings among quarks of pions. Nevertheless, we have shown [8] [9] other an equivalent way can also calculate the mass defect: applying the matrix $A_{i j}$ of mass defects $\left(\Delta m_{i}\right)$ of particle $X$. The matrix $A_{i j}$ is so defined, Table 1.

Table 1. The couplings with the interaction of quarks.

\begin{tabular}{ccccc}
\hline$\Delta m(X)$ & $q_{1}$ & $q_{2}$ & $\ldots$ & $q_{n}$ \\
\hline$q_{1}$ & $\Delta m\left(q_{1}, q_{1}\right)_{\oplus}^{R}$ & $\Delta m\left(q_{1}, q_{2}\right)_{\oplus}^{G}$ & $\ldots$ & $\Delta m\left(q_{1}, q_{n}\right)_{\oplus}^{B}$ \\
$q_{2}$ & $\Delta m\left(q_{2}, q_{1}\right)_{\oplus}^{G}$ & $\Delta m\left(q_{2}, q_{2}\right)_{\oplus}^{R}$ & $\ldots$ & $\Delta m\left(q_{2}, q_{n}\right)_{\oplus}^{G}$ \\
$\ldots$ & $\ldots$ & $\ldots$ & $\ldots$ & $\ldots$ \\
$q_{n}$ & $\Delta m\left(q_{n}, q_{1}\right)_{\oplus}^{B}$ & $\Delta m\left(q_{n}, q_{2}\right)_{\oplus}^{G}$ & $\ldots$ & $\Delta m\left(q_{n}, q_{n}\right)_{\oplus}^{R}$ \\
\hline
\end{tabular}

Where $q_{i}$ is the base particles (here we have quarks) which compose the particle 
$X$. Besides, it is $\Delta m\left(q_{i}, q_{j}\right)_{\oplus}^{R, B, G}=\Delta m\left(q_{i} \oplus q_{j}\right)^{R, B, G}$. The superscripts $(R, G, B)$ are colors which point out positive mass defects $(G)$ and negative $(B)$, while $(R)$ the elements of the diagonal to values positive or negative in according to specific cases.

\section{The Nucleon Masses}

\subsection{The Mass of the Proton (938.27 MeV)}

In light mesons, we highlighted a mass spectrum built employing of lattices of base pions $\{\pi\}$ and quarks $\{d, \underline{d}\}$ [9]. In the Baryons of the first octet, using a quark lattice but not pions we build the mass spectrum because the Baryons are built with three component quarks. Therefore, the fundamental element is not a couple of quarks but the individual quark and its possible coupling. The coupling of quarks with interpenetration will be expressed by $\underline{\otimes}$-operator: $(q \underline{\otimes} q \underline{\otimes}$ $q)$.The product $[u \underline{\otimes} d \otimes \underline{u}]$ imply the combination of all possible configurations between quarks $(u, d, u)$.

Recall $[\underline{\otimes}=\otimes U \oplus]$, see ref. [7] [8] [9], where the $\otimes$-operation expresses the interpenetration between quarks while the $\oplus$-operation expresses them eventual interactions through gluons and photons. Nevertheless, we incorporate the elastic tensions of binding gluons into opportune mass values, see the quarks' masses $\left[m\left(u_{\pi}\right), m\left(d_{\pi}\right)\right]$ in the pion. The structure equation needs to describe, thus, the interpenetration di each quark $\left(q_{i}\right)$ with another quark $\left(q_{j}\right)$ and with the pair of two "bonded" quarks $\left(q_{j} \oplus q_{k}\right)$. In this way, the proton will be a "superposition" of all possible combinations made means $(\underline{\otimes} \equiv \otimes U \oplus)$ with three quarks $(u, d, u)$. These coupling combinations can be the followings:

$$
\left[\left(u_{1} d\right),\left(u_{1} u_{2}\right),\left(d u_{2}\right)\right]_{A 1},\left[u_{1}\left(d u_{2}\right), d\left(u_{1} u_{2}\right), u_{2}\left(u_{1} d\right)\right]_{A 2}
$$

The property of operation $(\underline{\otimes})$ which describes the combinations of three quarks is:

$$
[A \otimes \underline{B} \otimes C]=\{[(A) \otimes(B \oplus C)] \oplus[(B) \otimes(C \oplus A)] \oplus[(C) \otimes(A \oplus B)]\}
$$

The geometric representation of proton admits two configurations, Figure 3.

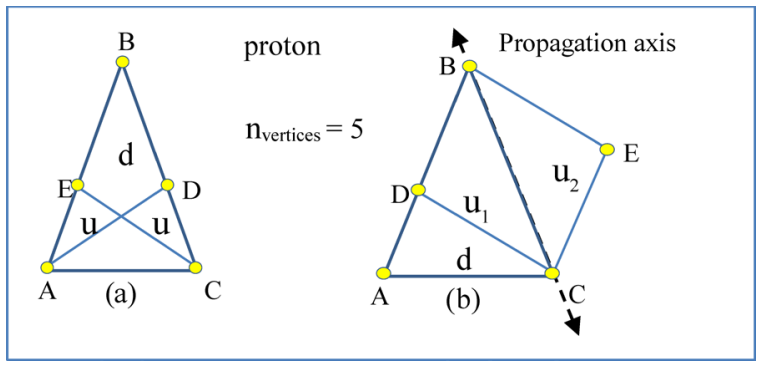

Figure 3. Proton configurations.

Note the configuration $1(\mathrm{~b})$ shows an electric charge equal to one, along the propagation side $\mathrm{BC}$.

Also if we give a structure of coupling oscillators to the proton, the same one 
needs to associate a wave function to the proton and, thus, a field of quantum oscillators, expressed by annihilation operator (a) and creation $\left(a^{+}\right)$. The representative quanta of the proton propagate along the axis passing through $\mathrm{B}$ and $\mathrm{C}$ (see Figure 3); this axis consists of a chain of quantum oscillators belonging to the diagonal of junction of a lattice of pairs $[(d, \underline{d}),(u, \underline{u})]$, see later the neutron and the figure (11) in conclusion. The possibility of having two configurations is coherent to that in ref. [11] a team of physicists suggested: the fundamental building unit proton can alter its structure under certain circumstances. Scientists are now performing experiments to show that the structure of protons can change inside the nucleus under certain conditions (see the two configurations in Figure 3). We think, see the postulates of QM, that the two configurations represent two possible states (eigenstates) which must be observables. The a-configuration could be more present in protons bound to neutron in the nucleus; instead, the $b$-configuration could be more present in free protons. In interactions $(p+p)$ we can detect, see the b-configuration, two hadronic jets, in opposite directions, but along the side BC. However, we can also have three jets: two, in opposite directions along the axis $\mathrm{BC}$, while the other along the side $\mathrm{AB}$ (a-configuration).

Let us go to determining the structure equation of the proton. Through geometric structure, see Figure 1 and Figure 3, one can deduce that a quark $\left(q_{i}\right)$ interpenetrates the others two quark $\left(q_{j}, q_{k}\right)$ in reciprocal interaction; this aspect needs to occur for each quark. Using Equation (7), we will have:

$$
\begin{aligned}
& {\left[u_{1} \otimes d \otimes u_{2}\right]} \\
& =\left\{\left[\left(u_{1}\right) \otimes\left(d \oplus u_{2}\right)\right]_{A_{1}} \oplus\left[(d) \otimes\left(u_{2} \oplus u_{1}\right)\right]_{A_{2}} \oplus\left[\left(u_{2}\right) \otimes\left(u_{1} \oplus d\right)\right]_{A_{3}}\right\}_{A_{3}}
\end{aligned}
$$

This relation tells us that the quarks reciprocally interpenetrate, but at the same time, they interact between them. Therefore, the structure equation of the proton is:

$$
(p)=\left(\left\{3 \kappa_{p}\left[u_{1} \otimes d \otimes u_{2}\right]\right\}\right)
$$

where the number 3 point out the 3-dimensionality of a baryon (three free degrees) while to the mesons we assign a 2-dimensionality or two free degrees. The $\kappa_{p}$ coefficient is in connection to the global elasticity of the system of quantum oscillators $(\mathrm{IQuO})$ of the geometric structure of the quarks into nucleons: we highlight that the increase of the number of vertices in a geometric structure makes increase the elastic tension between the quantum oscillators of structure.

The $\oplus$-operation in eq. 8 not cancels the no-locality of superposition of three states $\left(\Psi_{A}\right)$ associated with $A_{i}$-components (only an outer observation or interaction could reduce the $(\Psi))$. The electric charge is, instead, already connected to an eigenstate of the wave function, relatives to its phase [19] [20]. Using Equation (8), we can have:

$$
\begin{aligned}
p & =3 \kappa_{p}\left\{\left[u_{1} \otimes\left(d \oplus u_{2}\right)\right]_{A_{1}} \oplus\left[d \otimes\left(u_{2} \oplus u_{1}\right)\right]_{A_{2}} \oplus\left[u_{2} \otimes\left(u_{1} \oplus d\right)\right]_{A_{3}}\right\} \\
& =\left\{3 \kappa_{p}\left[p_{a} \oplus p_{b} \oplus p_{c}\right]\right\}
\end{aligned}
$$

where $(a, b, c)$ are the indices of the following relations: 


$$
\begin{aligned}
& p_{a}=\left[u_{1} \otimes\left(d \oplus u_{2}\right)\right]_{a}=\left[\left(u_{1} \otimes d\right) \oplus\left(u_{1} \otimes u_{2}\right)\right]_{a} \\
& p_{b}=\left[d \otimes\left(u_{2} \oplus u_{1}\right)\right]_{b}=\left[\left(d \otimes u_{2}\right) \oplus\left(d \otimes u_{1}\right)\right]_{b} \\
& p_{c}=\left[u_{2} \otimes\left(u_{1} \oplus d\right)\right]_{c}=\left[\left(u_{2} \otimes u_{1}\right) \oplus\left(u_{2} \otimes d\right)\right]_{c}
\end{aligned}
$$

Now, we calculate partial mass of the proton, using the properties of operations $(\otimes, \oplus)$, see the tables in ref. [9] and Function $F_{m}$ in Equations (4) and (5). In first, it is:

$$
\begin{aligned}
& F_{m}\left(p_{a}\right)=m\left(p_{a}\right)=m\left[\left(u_{1} \otimes d\right) \oplus\left(u_{1} \otimes u_{2}\right)\right]_{a}=m\left(\left\langle u_{1}, d\right\rangle\right)+m\left(\left\langle u_{1}, u_{2}\right\rangle\right) \\
& F_{m}\left(p_{b}\right)=m\left(p_{b}\right)=m\left[\left(d \otimes u_{2}\right) \oplus\left(d \otimes u_{1}\right)\right]_{b}=m\left(\left\langle d, u_{2}\right\rangle\right)+m\left(\left\langle d, u_{1}\right\rangle\right) \\
& F_{m}\left(p_{c}\right)=m\left(p_{c}\right)=m\left[\left(u_{2} \otimes u_{1}\right) \oplus\left(u_{2} \otimes d\right)\right]_{c}=m\left(\left\langle u_{2}, u_{1}\right\rangle\right)+m\left(\left\langle u_{2}, d\right\rangle\right)
\end{aligned}
$$

With sum:

$$
\begin{aligned}
m\left(p_{a, b, c}\right) & =m\left(p_{a}\right)+m\left(p_{b}\right)+m\left(p_{c}\right) \\
& =2 m\left(\left\langle u_{1}, d\right\rangle\right)+2 m\left(\left\langle u_{2}, d\right\rangle\right)+2 m\left(\left\langle u_{1}, u_{2}\right\rangle\right)
\end{aligned}
$$

This because is $[A \otimes B]=[B \otimes A]$. Nevertheless, note the quarks masses $(u, d)$ of proton could be different to one of pion: $\left[m\left(u_{p}\right), m\left(d_{p}\right)\right] \neq\left[m\left(u_{\pi}\right), m\left(d_{\pi}\right)\right]$

The couplings $\left[\left[u_{1}\left(d u_{2}\right), d\left(u_{1} u_{2}\right), u_{2}\left(u_{1} d\right)\right]_{A 2}\right]$ in proton induces us to think that the pair $\left(u_{p}, d_{p}\right)$ needs considering with elastic tension more strong than pair $\left(u_{\pi}, d_{\pi}\right)$ :

$$
\left(u_{p}, d_{p}\right)=k_{p}\left(u_{\pi}, d_{\pi}\right),\left(k_{p}>0\right)
$$

We have already said that the elastic tension $(\kappa)$ between the IQuO of sides and the ones of vertices is function of them number; then, it is intuitive considering the parameter $\kappa$ given by the rapport between $n_{V}$ number vertices

$\left[\left(A(d), B(d), C(d), D\left(u_{1}\right), E\left(u_{2}\right)\right]\right.$ of geometric structure (in proton is $n_{V}=5$ ) and number of total $\left(n_{e l}\right)$ of the "coupling" vertices (elastic vertices) between two quarks:

- In b-config.: $\left[\left(B\left(d, u_{1}\right), C\left(d, u_{1}\right) ;\left(B\left(d, u_{2}\right), C\left(d, u_{2}\right) ;\left(B\left(u_{1}, u_{2}\right), C\left(u_{1}, u_{2}\right)\right] \Leftrightarrow\left(n_{e l_{v}}\right)\right.\right.\right.$ $=6$

- In a-config.: $\left[\left(B\left(d, u_{1}\right), C\left(d, u_{2}\right) ;\left(B\left(d, u_{2}\right) ; B\left(u_{1}, u_{2}, d\right)\right] \Leftrightarrow\left(n_{e l v}\right)=6\right.\right.$

Where $B\left(u_{1}, u_{2}, d\right)$ is a triple vertex. It follows: $\left[\kappa_{p}=n_{v} /\left(n_{e I_{v}}\right)=5 / 6\right]$. Then, by mass values of solutions of Equation (1), the proton mass is:

$$
\begin{aligned}
m(p) & =\left\{3 \kappa_{p}\left[m\left(p_{a}\right)+m\left(p_{b}\right)+m\left(p_{c}\right)\right]\right\} \\
& =3 \kappa_{p}\left[2 m\left(\left\langle u_{1}, d\right\rangle\right)+2 m\left(\left\langle u_{2}, d\right\rangle\right)+2 m\left(\left\langle u_{1}, u_{2}\right\rangle\right)\right] \\
& =3 \kappa_{p}\left[4 m(\langle u, d\rangle)+2 m\left(\left\langle u_{1}, u_{2}\right\rangle\right)\right] \\
& =3 \kappa_{p}[4(69.79)+2(53.31)] \mathrm{MeV}
\end{aligned}
$$

Having that $\left[\kappa_{p}=(5 / 6)\right]$, it follows:

$$
\begin{aligned}
m(p) & =3\left(\frac{5}{6}\right)[4 m(\langle u, d\rangle)+2 m(\langle u, u\rangle)] \\
& =[10(69.79)+5(53.31)] \mathrm{MeV}=(964.45) \mathrm{MeV}
\end{aligned}
$$

In the structure equation, one can identify the various defects of mass. The electromagnetic mass defects will be $\Delta m_{\gamma}\left(u_{p}, d_{p}\right)$ and $\Delta m_{\gamma}\left(u_{p}, u_{p}\right)$. 
We can admit that: $\Delta m_{\gamma}\left(q_{i}, q_{j}\right)_{p}=3 \kappa_{p} \Delta m_{\gamma}\left(q_{i}, q_{j}\right)_{\pi}$. Recall that [7]:

$$
\Delta m_{\gamma}\left(u_{\pi}, d_{\pi}\right)=m\left(\pi^{ \pm}\right)-m\left(\pi^{0}\right)=\Delta m\left(\varepsilon_{\pi^{ \pm}}(\gamma)\right)=(4.59) \mathrm{MeV} / c^{2}
$$

With

$$
\Delta m\left(\varepsilon_{\pi^{ \pm}}(\gamma)\right)=\frac{1}{2}\left[m_{\gamma}(u)+m_{\gamma}(d)\right]=m_{\gamma}(\langle u, d\rangle)
$$

where $m_{\gamma}(q)$ is the electromagnetic mass of quark, that is the quark mass generating photons in annihilation processes. Then, $m_{\gamma}(q)$ represents the free mass of quarks or, also, the bare mass, see Equation (2). We will have here:

$$
\left\{\begin{array}{c}
m_{\gamma}(u)=(3.51) \mathrm{MeV} / c^{2}=\Delta m_{\gamma}(u, u) \\
m_{\gamma}(d)=(5.68) \mathrm{MeV} / c^{2}=\Delta m_{\gamma}(d, d)
\end{array}\right\}
$$

where we have so calculated $\Delta m_{\gamma}(u, u)$ and $\Delta m_{\gamma}(d, d)$. Note that these values are coincident with the values of the superior extremes of mass interval reported in experimental literature [3] [15]. These experimental values tell us that the masses of quarks found experimentally are the electromagnetic masses. Instead, the masses used in Equation (1) are the gluons masses originated by elastic tension of gluon nature, because the couplings between quantum oscillators building the geometric structure of quarks are constituted by junction gluons (remember $\left.\left[m_{\pi}(u), m_{\pi}(d)\right]\right)$. So, using the mass values $\left[m_{p}(u), m_{p}(d)\right]$, we incorporate the mass defects relative to gluon interactions between quarks consequently.

We could calculate the global mass defect $\Delta m_{\gamma}(p)$ in two equivalent way [8] [9]:

1) Using the $F_{\Delta m}$-function

2) Using the matrix $A_{i j}$ of the mass defects $\Delta m_{q}$

Here, we choose the matrix $A_{i j}$ of mass defects. Then, we build the matrix $A_{i j}$ of $\Delta m_{\gamma}(q)$ of all couplings with interpenetration between quarks $\left[\left(q_{i} \otimes q_{j}\right)\right]$, which compose the structure equation in p-nucleon and interacting with other quark, $\left[\left(q_{i} \otimes q_{j}\right)_{\oplus}\right]$.

If the interpenetrating quark pair interacts to other quark, $\left[\left(q_{i} \otimes q_{j}\right) \oplus q_{j}\right]$, then it is: $\left[\left(q_{i} \otimes q_{j}\right)_{\oplus}\right] \neq\left[\left(q_{j} \otimes q_{i}\right)_{\oplus}\right]$. This aspect gives the no-commutation of field operators $\left(q_{i}, q_{j}\right)$. The matrix is, see Table 2.

Table 2. The couplings with the interaction of quarks in the proton.

\begin{tabular}{cccc}
\hline$\Delta \boldsymbol{m}^{*}(\boldsymbol{p})$ & $\boldsymbol{u}_{1}$ & $\boldsymbol{d}_{1}$ & $\boldsymbol{u}_{2}$ \\
\hline$u_{1}$ & $\Delta m\left(u_{1}, u_{1}\right)_{\oplus}^{R}$ & $\Delta m\left(u_{1}, d_{1}\right)_{\oplus}^{G}$ & $\Delta m\left(u_{1}, u_{2}\right)_{\oplus}^{B}$ \\
$d_{1}$ & $\Delta m\left(d_{1}, u_{1}\right)_{\oplus}^{G}$ & $\Delta m\left(d_{1}, d_{1}\right)_{\oplus}^{R}$ & $\Delta m\left(d_{1}, u_{2}\right)_{\oplus}^{G}$ \\
$u_{2}$ & $\Delta m\left(u_{2}, u_{1}\right)_{\oplus}^{B}$ & $\Delta m\left(u_{2}, d_{1}\right)_{\oplus}^{G}$ & $\Delta m\left(u_{2}, u_{2}\right)_{\oplus}^{R}$ \\
\hline
\end{tabular}

For calculating the mass defect, we sum all mass defects relative to elements of the matrix. We are turning to Equations (16)-(18): 


$$
\begin{aligned}
\Delta m^{*}(p)= & {\left[4 \Delta m\left((u \otimes d)_{\oplus}\right)\right]_{G}+\left[2 \Delta m\left(\left(u_{1} \otimes u_{2}\right)_{\oplus}\right)\right]_{B} } \\
& +\left[\Delta m\left(\left(u_{1} \otimes u_{1}\right)_{\oplus}\right)+\Delta m\left(\left(u_{2} \otimes u_{2}\right)_{\oplus}\right)+\Delta m\left((d \otimes d)_{\oplus}\right)\right]_{R} \\
= & \left\{\left[(18.36)_{G}-(7,02)_{B}\right] \mathrm{MeV}+\left[\left(R\left(u_{1}\right)+R\left(u_{2}\right)+R(d)\right)_{\oplus}\right]_{R}\right\} \\
= & (11.34) \mathrm{MeV}+R\left(q_{i}\right)
\end{aligned}
$$

Recall the $\Delta m_{\gamma}\left(q_{i}, q_{j}\right)_{p}>0$, while $\Delta m_{\gamma}\left(q_{i}, q_{i}\right)_{p}<0$.

For finding the values of mass defects $R\left(u_{i}\right)$ and $R(d)$, we highlight the spin values of three quarks $(u, u, d)$ and we give the coefficients $\left(r_{i}\right)$ to function $R$ of the mass defects:

$$
\begin{aligned}
R_{\oplus} & =\left[\left(R\left(u_{1}\right)+R\left(u_{2}\right)+R(d)\right)\right] \\
& =\left[\left(r_{1} \Delta m\left(u_{(\uparrow)} \otimes u_{(\uparrow)}\right)_{u_{1}}+r_{2} \Delta m\left(u_{(\downarrow)} \otimes u_{(\downarrow)}\right)_{u_{2}}+r_{3} \Delta m\left(d_{(\uparrow)} \otimes d_{(\uparrow)}\right)_{d}\right)_{\oplus}\right]_{R}
\end{aligned}
$$

The arrows of the subscripts in terms of $(u)$ and $(d)$ point out the spin, see Equation (3).

In ref. [8] [9] to elements in red of $A_{i j}$ matrix in mesons, we gave the values of $(1,15) \mathrm{MeV}$. In the proton, we assume the same value (see the couplings $\left(q_{p} q_{j}\right)$ as in meson), also if we can admit some corrections to this value. To give a numerical value of mass defect to the terms $\left(q_{i} \otimes q_{i}\right)_{R}$ would imply the interpenetration of a quark with itself. Now, we need to give a plausible explanation for this paradoxical aspect. A quark in rotation around to an axis implies different configurations of itself to the time flow. Recall the QM admit a not space locality, see the superposition of states in the experiments of the undulatory mechanics with two fissures. If the time is a coordinate equivalent to one of space (see the relativity), then we need also talk about not time locality (note that we are also talking about a time operator in QM). In this way we can admit some kind of time superposition of a particle with itself, which, however, must not violate the energy conservation: so, a particle cannot interfere negatively with itself (surely, if the particle is free). The positive superposition in time with itself might imply the interpenetration with itself of a particle. This interpenetration might have a dynamic component with a negative mass defect. All this is possible only in proper space rotations (spin) and not in space translations. This quantum aspect of proper rotation and of not time locality of quarks leads us to conjecture that the elements in red color (in diagonal) of the mass defect matrix can represent the kinetic energy of rotation of quarks around to the side $\mathrm{AB}$, see Figure 2. Therefore, we can have $\Delta m\left(q_{i} \otimes q_{i}\right)_{\oplus} \neq 0$. To this orbital kinetic energy, we associate a mass defect negative $\Delta m_{\text {Kin }}<0$. Recall, in this way, the influence of the spin of a particle in them interactions. About the proton spin, see ref. [17] [18], we can have:

$$
\begin{aligned}
s_{p}= & \left\{\begin{array}{c}
s_{t}(q)=\left[(\uparrow)_{u_{1}},(\downarrow)_{u_{2}},(\uparrow, \downarrow)_{d_{1}}\right] \equiv s_{q}\left(u_{1}\right)+s_{q}\left(u_{2}\right)+s_{q}\left(d_{1}\right)=s_{q}\left(d_{1}\right)= \pm(1 / 2) \\
s_{t}(l)=\left[(\uparrow)_{u_{1}},(\downarrow)_{u_{2}},(\uparrow, \downarrow)_{d_{1}}\right] \equiv s_{l}\left(u_{1}\right)+s_{l}\left(u_{2}\right)+s_{l}\left(d_{1}\right)=s_{l}\left(d_{1}\right)= \pm(1 / 2) \\
s_{t}(g)=\left[(\downarrow)_{u_{1}},(\uparrow)_{u_{1}},(\downarrow, \uparrow)_{d_{1}}\right] \equiv s_{g}\left(u_{1}\right)+s_{g}\left(u_{2}\right)+s_{g}\left(d_{1}\right)=s_{g}\left(d_{1}\right)=\mp(1 / 2)
\end{array}\right\} \\
= & s_{q}\left(d_{1}\right)= \pm(1 / 2)
\end{aligned}
$$


Note that $[s(I)+s(g)=0]$ and $\left[\left(s_{\text {tot }}(q)= \pm 1 / 2, s_{\text {tot }}(I)= \pm 1\right),\left(s_{\text {tot }}(g)=\mp 1\right)\right]$ : the orbital spin is always opposite to gluon spin. From Equaiton (21), the contribution of the quarks' spin to total spin of proton is (1/3), see the literature. Besides, all quarks are free in their orbital movement, see the literature, even if there is a binding connection between $\left(q_{p} q_{j}\right)$. Then, we will have:

$$
\begin{aligned}
& \left(R\left(u_{1}\right)+R\left(u_{2}\right)+R(d)\right)^{\prime} \\
& \left.\left.=\left[\left(r_{1}^{\prime} \Delta m^{\prime}\left(u_{(\uparrow)} \otimes u_{(\uparrow)}\right)\right)_{u_{1}}+r_{2}^{\prime} \Delta m^{\prime}\left(u_{(\downarrow)} \otimes u_{(\downarrow)}\right)_{u_{2}}+r_{3}^{\prime} \Delta m^{\prime}\left(d_{(\uparrow)} \otimes d_{(\uparrow)}\right)\right)_{d}\right)\right] \\
& =\left[\left((-1)_{u_{1}}+(+1)_{u_{2}}+(-1)_{d}\right)\right]_{R}(1.15) \mathrm{MeV}=\left[\left(0_{u, u}-1_{d}\right)\right]_{R}(1.15) \mathrm{MeV}
\end{aligned}
$$

where we have calculated the eigenvalues of $\left[R(q)=r \Delta m\left(q_{i} \otimes q_{i}\right)_{\oplus}\right]$ :

$R^{\prime}(q)=r^{\prime}\left[\Delta m^{\prime}\left(q_{i} \otimes q_{i}\right)\right]$ with eigenvalues $r^{\prime}= \pm 1$ and $\left[\Delta m^{\prime}\left(q_{i} \otimes q_{i}\right)\right]=(1.15) \mathrm{MeV}$

Exactly, we have

1) $r^{\prime} \Delta m^{\prime}\left(u_{1} \otimes u_{1}\right)=-r^{\prime} \Delta m^{\prime}\left(u_{2} \otimes u_{2}\right)^{\prime} \quad$ because the spins are opposite

2) $r^{\prime} \Delta m^{\prime}\left(u_{1} \otimes u_{1}\right)^{\prime}=-r^{\prime} \Delta m^{\prime}(d \otimes d)^{\prime}$ because quarks are reciprocally in relative movement

Then we have:

$$
\Delta m^{*}(p)=\left\{[(11.34)]_{G B}-\left[(1.15)_{R}\right]\right\} \mathrm{MeV}=(10.19) \mathrm{MeV}
$$

Nevertheless, the value $(1,15) \mathrm{MeV}$ is only approximate, see ref [9]. With this approximation we obtain the total mass defect, being explicit the elasticity parameter $\kappa_{p}$, see Equation (15):

$$
\Delta m(p)=3 \kappa_{p}\left[\Delta m^{*}(p)\right]=\left(\frac{5}{2}\right)[(10.19)] \mathrm{MeV}=(25.48) \mathrm{MeV}
$$

Then, the total mass of the proton is:

$$
m_{\text {tot }}(p)=m(p)-\Delta m_{\gamma}(p)=[(964.45)-(25.48)] \mathrm{MeV}=(938.97) \mathrm{MeV}
$$

Value very next to the experimental.

The state with $\left[\left(s_{t o t}\right)_{q}= \pm 1 / 2\right]$ is the one of proton, while the state with $\left[s_{t o t}(q)\right.$ $= \pm 3 / 2]$ is the resonance $N^{+}(3 / 2)_{(1520) \mathrm{MeV}}:(p+\gamma) \rightarrow N^{+} \rightarrow(p+\gamma)$, see ref. [3] in pag. 1660.

$$
s_{N^{+}}=\left\{\begin{array}{c}
s_{t}(q)=\left[(\uparrow, \downarrow)_{u_{1}},(\uparrow, \downarrow)_{u_{2}},(\uparrow, \downarrow)_{d_{1}}\right] \equiv s_{q}\left(u_{1} d_{1} u_{2}\right)= \pm(3 / 2) \\
s_{t}(l)=\left[(\uparrow, \downarrow)_{u_{1}},(\uparrow, \downarrow)_{u_{2}},(\uparrow, \downarrow)_{d_{1}}\right] \equiv s_{l}\left(d_{1}\right)= \pm 3 \\
s_{t}(g)=\left[(\downarrow, \uparrow)_{u_{1}},(\downarrow, \uparrow)_{u_{1}},(\downarrow, \uparrow)_{d_{1}}\right] \equiv s_{g}\left(d_{1}\right)=\mp 3
\end{array}\right\}=s_{q}= \pm(3 / 2)
$$

In the next paper, we will show the structure equation of resonance $N^{+}(3 / 2)$ and its calculation of mass.

\subsection{The Neutron}

We might think to follow geometric structures of the neutral state with three 
quarks $(d, u, d)$ (Figure 4$)$ :

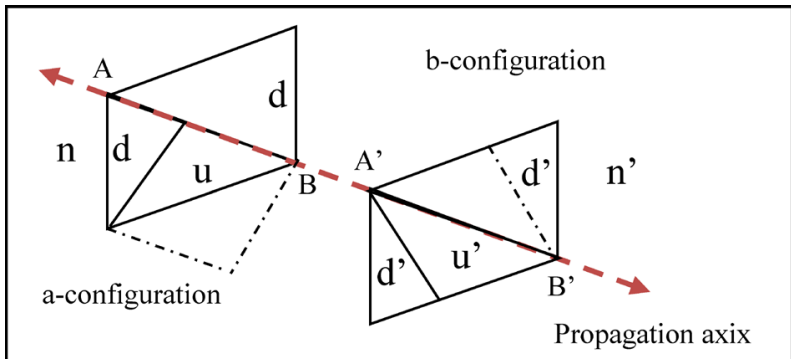

Figure 4. The two base configurations of the neutron.

Note, in the b-configuration, along the diagonal (A'B'), the sum of the electric charge of quarks is zero. In previous works [9] for calculating the mass values of mesons, we needed to admit the presence of a background lattice of pairs $\{d, \underline{d}\}$, which comes included in the structure equation of mesons. The same it also occurs in the light neutral nucleon, where a couple $(d, \underline{d})$, belonging to background lattice, is being in "loan" for being incorporated in them structure: then, it needs add, into calculations of them mass, a quarks' pair $(d, \underline{d})$. Therefore, the structure equation could be:

$$
(n)=3 \kappa_{n}\left(\left\{(d \otimes \underline{d})_{A} \otimes\left[d_{1} \otimes u \otimes d_{2}\right]_{B}\right\}\right)
$$

With configuration, see Figure 5:

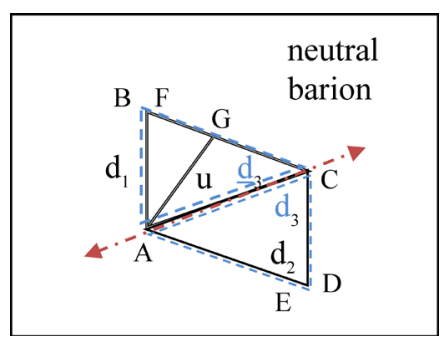

Figure 5. The neutron configuration.

The $\kappa_{n}$ is the elasticity coefficient modulating the masses of quarks $(u, d)$ respect to one of pion $\left(u_{m}, d_{\pi}\right):\left[m\left(u_{n}, d_{n}\right)=\kappa_{n} m\left(u_{\pi}, d_{\pi}\right)\right]$

The vertices number $[A, B, C, D, F, G, E]$ is $n_{V}=7$, while the number of total $\left(n_{e l}\right)$ of the "coupling" elastic vertices of quarks is:

[( $\left.A\left(d_{1}, u\right), C\left(d_{1}, u\right) ; A\left(d_{1}, d_{2}\right), C\left(d_{1}, d_{2}\right) ; A\left(d_{2}, u\right), C\left(d_{2}, u\right) ; A\left(d_{3}, \underline{d}_{3}\right), C\left(d_{3}, \underline{d}_{3}\right)\right]$ or number 8

We find: $\left[\kappa_{n}=n_{v} /\left(n_{e l}\right)=7 / 8\right]$.

Recall, the increasing of number of vertices in a geometric structure makes increase the elastic tension between the quantum oscillators of structure. Processing the structure Equation (27), we will have:

$$
\begin{aligned}
n & =3 \kappa_{n}\left\{[(d \otimes \underline{d})]_{A} \otimes\left\{\left\{\left[d_{1} \otimes\left(u \oplus d_{2}\right)\right]_{B_{1}} \oplus\left[u \otimes\left(d_{2} \oplus d_{1}\right)\right]_{B_{2}} \oplus\left[d_{2} \otimes\left(d_{1} \oplus u\right)\right]_{B_{3}}\right\}\right\}\right. \\
& =3 \kappa_{n}\left\{[(d \otimes \underline{d})]_{A} \otimes\left[N_{a} \oplus N_{b} \oplus N_{c}\right]_{B}\right\}
\end{aligned}
$$


The mass calculation uses $F_{m}$-function. In this case, with the presence of the pair $(d, \underline{d})$, we project the operation $(A \underline{\otimes} B)$ in $F_{m}$-function space:

$$
\left[\otimes_{F m}=(\otimes \mathrm{U} \oplus)_{F m}=\oplus_{\otimes}\right]
$$

That is the operation $\left(\oplus_{\otimes}\right)$ point out interaction with interpenetration of components. Then, it is:

$$
\begin{aligned}
m(n)= & 3 \kappa_{n} F_{m}\left([(d \otimes \underline{d})]_{A} \otimes\left[n_{a} \oplus n_{b} \oplus n_{c}\right]_{B}\right) \\
= & 3 \kappa_{n} m\left([ ( d \otimes \underline { d } ) ] _ { A } \oplus _ { \otimes } \left\{\left[d_{1} \otimes\left(u \oplus d_{2}\right)\right]_{B_{1}}\right.\right. \\
& \left.\left.\oplus\left[u \otimes\left(d_{2} \oplus d_{1}\right)\right]_{B_{2}} \oplus\left[d_{2} \otimes\left(d_{1} \oplus u\right)\right]_{B_{3}}\right\}\right)_{\otimes} \\
= & 3 \kappa_{n}\left\{m(d \otimes \underline{d})_{A}+m\left(\left\{\left[d_{1} \otimes\left(u \oplus d_{2}\right)\right]_{B_{1}}\right.\right.\right. \\
& \left.\left.\left.\oplus\left[u \otimes\left(d_{2} \oplus d_{1}\right)\right]_{B_{2}} \oplus\left[d_{2} \otimes\left(d_{1} \oplus u\right)\right]_{B_{3}}\right\}\right)\right\}_{\otimes} \\
= & {\left[3 \kappa_{n} m(d)\right]_{A}+\left\{3 \kappa_{n}\left[m\left(n_{a}\right)+m\left(n_{b}\right)+m\left(n_{c}\right)\right]\right\}_{B} } \\
= & 3 \kappa_{n} m(n)_{A}+3 \kappa_{n} m(n)_{B}
\end{aligned}
$$

With

$$
\begin{aligned}
& m\left(n_{a}\right)=m\left[\left(d_{1} \otimes u\right) \oplus\left(d_{1} \otimes d_{2}\right)\right]_{a}=m\left(\left\langle d_{1}, u\right\rangle\right)+m\left(\left\langle d_{1}, d_{2}\right\rangle\right) \\
& m\left(n_{b}\right)=m\left[\left(u \otimes d_{2}\right) \oplus\left(u \otimes d_{1}\right)\right]_{b}=m\left(\left\langle u, d_{2}\right\rangle\right)+m\left(\left\langle u, d_{1}\right\rangle\right) \\
& m\left(n_{c}\right)=m\left[\left(d_{2} \otimes d_{1}\right) \oplus\left(d_{2} \otimes u\right)\right]_{c}=m\left(\left\langle d_{2}, d_{1}\right\rangle\right)+m\left(\left\langle d_{2}, u\right\rangle\right)
\end{aligned}
$$

The state with spin $s=1 / 2$ is the neutron, see Figure 5 .

If the neutron spin is $(1 / 2)$ and $u$-quark rotates around axis AC, then the two $d$-quarks have opposite spin to it. Recall the $(d, \underline{d})_{\gamma}$ pair is at spin $s=1$; thus the two couples $\left[(d, d),(d, \underline{d})_{\gamma}\right]$ have an overall zero spin. It follows:

$$
s_{n}=\left\{\begin{array}{c}
s_{t}(q)=\left[(\downarrow \downarrow)_{\left(d_{1}, d_{2}\right)},(\uparrow, \downarrow)_{u_{1}}\right],\left[(\uparrow \uparrow)_{(d, \underline{d})_{\gamma}}\right] \equiv\left[s_{\gamma}(d, \underline{d})+s_{q}\left(d_{1}, d_{2}\right)\right]+s_{q}\left(u_{1}\right)= \pm(1 / 2) \\
s_{t}(l)=\left[(\downarrow \downarrow)_{\left(d_{1}, d_{2}\right)},(\uparrow, \downarrow)_{u_{1}}\right],\left[(\uparrow \uparrow)_{(d, \underline{d})_{\gamma}}\right] \equiv s_{l}\left(u_{1}\right)= \pm(1 / 2) \\
s_{t}(g)=\left[(\uparrow \uparrow)_{\left(d_{1}, d_{2}\right)},(\downarrow, \uparrow)_{u_{1}}\right],\left[(\downarrow \downarrow)_{(d, \underline{d})_{\gamma}}\right] \equiv s_{g}\left(u_{1}\right)=\mp(1 / 2)
\end{array}\right\}
$$

In all two cases, the two quarks $\left(d_{1}, d_{2}\right)$ cannot interpenetrate each other, because

They have parallel spins: then, it is $\left(d_{1} \otimes d_{2}\right) \equiv 0$ and, thus, $\left[m\left(d_{1} \otimes d_{2}\right)=0\right]$, see Table 2 in ref [9]. Being $\left[m\left(d_{1} \otimes d_{2}\right)=0\right]$ it follows, see Equation (30):

$$
\begin{aligned}
& m\left(n_{a}\right)=m\left[\left(d_{1} \otimes u\right)\right]_{a}=m\left(\left\langle d_{1}, u\right\rangle\right) \\
& m\left(n_{b}\right)=m\left[\left(u \otimes d_{2}\right) \oplus\left(u \otimes d_{1}\right)\right]_{b}=m\left(\left\langle u, d_{2}\right\rangle\right)+m\left(\left\langle u, d_{1}\right\rangle\right) \\
& m\left(n_{c}\right)=m\left[\left(d_{2} \otimes u\right)\right]_{c}=m\left(\left\langle d_{2}, u\right\rangle\right)
\end{aligned}
$$


Thus:

$$
m\left(n_{a, b, c}\right)_{B}=\left[m\left(n_{a}\right)+m\left(n_{b}\right)+m\left(n_{c}\right)\right]_{B}=\left[2 m\left(\left\langle d_{1}, u\right\rangle\right)+2 m\left(\left\langle u, d_{2}\right\rangle\right)\right]_{B}
$$

and

$$
\begin{aligned}
m(n)_{B} & =\left\{\left[m\left(n_{a}\right)+m\left(n_{b}\right)+m\left(n_{c}\right)\right]\right\}=\left[2 m\left(\left\langle d_{1}, u\right\rangle\right)+2 m\left(\left\langle u, d_{1}\right\rangle\right)\right] \\
& =[4(69.79)] \mathrm{MeV}=(279.16) \mathrm{MeV}
\end{aligned}
$$

It follows

$$
\begin{aligned}
m(n) & =3 \kappa_{n}\left[m(n)_{A}+m(n)_{B}\right]=3 \kappa_{n}\left[m(d)+m(n)_{B}\right] \\
& =3 \kappa_{n}[(86.26)+(279.16)] \mathrm{MeV} \\
& =3\left(\frac{7}{8}\right)(365.42) \mathrm{MeV}=(959.23) \mathrm{MeV}
\end{aligned}
$$

As in the proton, also here we insert the interpenetration of a quark with itself. This last aspect comes highlighted by the matrix $A_{i j}$ We build the matrix $A_{i j}$ of all couplings with interpenetration between quarks $\left[\left(q_{i} \otimes q_{j}\right)\right]$, which compose the structure equation in n-nucleon and interacting with other quark, $\left[\left(q_{i} \otimes\right.\right.$ $\left.\left.q_{j}\right)_{\oplus}\right]$. As in proton, it is: $\left[\left(q_{i} \otimes q_{j}\right)_{\oplus}\right] \neq\left[\left(q_{j} \otimes q_{i}\right)_{\oplus}\right]$.

The matrix also admits the elements $A_{i i} \neq 0$. Then, the matrix is, see Table 3.

Table 3. The couplings with the interaction of quarks in a neutron.

\begin{tabular}{cccccc}
\hline$\Delta \boldsymbol{m}^{*}(\boldsymbol{n})$ & $\boldsymbol{d}_{1}$ & $\boldsymbol{u}_{1}$ & $\boldsymbol{d}_{2}$ & $\boldsymbol{d}_{3}$ & $\underline{\boldsymbol{d}}_{4}$ \\
\hline$d_{1}$ & $\left(d_{1} d_{1}\right)^{R}$ & $\left(d_{1} u_{1}\right)^{G 1}$ & $\left(d_{1} d_{2}\right)^{B 1}$ & $\left(d_{1} d_{3}\right)^{B 1}$ & $\left(d_{1} d_{4}\right)^{G 2}$ \\
$u_{1}$ & $\left(u_{1} d_{1}\right)^{G 1}$ & $\left(u_{1} u_{1}\right)^{R}$ & $\left(u_{1} d_{2}\right)^{G 1}$ & $\left(u_{1} d_{3}\right)^{G 1}$ & $\left(u_{1} \underline{d}_{4}\right)^{B 2}$ \\
$d_{2}$ & $\left(d_{2} d_{1}\right)^{B 1}$ & $\left(d_{2} u_{1}\right)^{G 1}$ & $\left(d_{2} d_{2}\right)^{R}$ & $\left(d_{2} d_{3}\right)^{B 1}$ & $\left(d_{2} d_{4}\right)^{G 2}$ \\
$d_{3}$ & $\left(d_{3} d_{1}\right)^{B 1}$ & $\left(d_{3} u_{1}\right)^{G 1}$ & $\left(d_{3} d_{2}\right)^{B 1}$ & $\left(d_{3} d_{3}\right)^{R}$ & $\left(d_{3} d_{4}\right)^{G 2}$ \\
$\underline{d}_{4}$ & $\left(\underline{d}_{4} d_{1}\right)^{G 2}$ & $\left(\underline{d}_{4} u_{1}\right)^{B 2}$ & $\left(\underline{d}_{4} d_{2}\right)^{G 2}$ & $\left(\underline{d}_{4} d_{3}\right)^{G 2}$ & $\left(\underline{d}_{4} \underline{d}_{4}\right)^{R}$ \\
\hline
\end{tabular}

Here we have omitted the subscript $(\oplus)$, see Table 1 in the proton. The mass defect is:

$$
\begin{aligned}
\Delta m^{*}(n)= & {\left[\Delta m(6(u \otimes d))_{G 1}+\Delta m(6(\underline{d} \otimes d))_{G 2}\right] } \\
& +\left[\Delta m\left(6\left(d_{i} \otimes d_{j}\right)\right)_{B 1}+2 \Delta m((u \otimes \underline{d}))_{B 2}\right] \\
& +\left[2 \Delta m\left(\left(d_{i} \otimes d_{i}\right)_{1,2}\right)+\Delta m\left(\left(d_{i} \otimes d_{i}\right)_{\gamma}\right)\right. \\
& \left.+\Delta m\left(\left(\underline{d}_{i} \otimes \underline{d}_{i}\right)_{\gamma}\right)+\Delta m\left(\left(u_{i} \otimes u_{i}\right)\right)\right]_{R}
\end{aligned}
$$

For the spin $(s=1 / 2)$, see the table of the neutron spin, we can have the term $R$ :

$$
\begin{aligned}
R^{\prime}(n)= & {\left[2 \Delta m\left(\left(d_{i} \otimes d_{i}\right)_{1,2}\right)+\Delta m\left(\left(d_{i} \otimes d_{i}\right)_{\gamma}\right)\right.} \\
& \left.+\Delta m\left(\left(\underline{d}_{i} \otimes \underline{d}_{i}\right)_{\gamma}\right)+\Delta m\left(\left(u_{i} \otimes u_{i}\right)\right)\right]_{R^{\prime}} \\
= & {\left[r_{1}^{\prime} \Delta m^{\prime}\left(d_{(\downarrow)} \otimes d_{(\downarrow)}\right)_{d_{1}}+r_{2}^{\prime} \Delta m^{\prime}\left(d_{(\downarrow)} \otimes d_{(\downarrow)}\right)_{d_{2}}+r_{3}^{\prime} \Delta m^{\prime}\left(d_{(\uparrow)} \otimes d_{(\uparrow)}\right)_{\gamma}\right.}
\end{aligned}
$$




$$
\begin{aligned}
& \left.+r_{4}^{\prime} \Delta m^{\prime}\left(\underline{d}_{(\uparrow)} \otimes \underline{d}_{(\uparrow)}\right)_{\gamma}+r_{5}^{\prime} \Delta m^{\prime}\left(u_{(\downarrow)} \otimes u_{(\downarrow)}\right)\right] \\
= & {\left[\left(-2_{\left(d_{1}, d_{2}\right)}+1_{\gamma}-1_{\gamma}-1_{u}\right)(1.15)_{R}\right] \mathrm{MeV}=\left[(-3)(1.15)_{R}\right] \mathrm{MeV} }
\end{aligned}
$$

Then, it is

$$
\begin{aligned}
\Delta m^{*}(n)= & {\left[\Delta m(6(u \otimes d))_{G 1}+\Delta m(6(\underline{d} \otimes d))_{G 2}\right] } \\
& +\left[\Delta m\left(6\left(d_{i} \otimes d_{j}\right)\right)_{B 1}+2 \Delta m((u \otimes \underline{d}))_{B 2}\right]+R(n) \\
= & {\left[6(4.59)_{G 1}+6(5.68)_{G 2}\right] \mathrm{MeV} } \\
& -\left[6(5.68)_{B 1}+2(4.59)_{B 2}\right] \mathrm{MeV}+\left[-(3.45)_{R}\right] \mathrm{MeV} \\
= & (14.91) \mathrm{MeV}
\end{aligned}
$$

The pair $(d, d)$ in the structure of neutron shields the electromagnetic interactions between quarks, therefore it weakens the mass defect. The presence of the pair $d$-quark along diagonal, see Figure 5, splits all mass defects, see ref. [9], then it follows:

$$
\Delta m^{* *}(n)=\left[\Delta m^{*}(n)\right]_{(d, \underline{d})}=\left(\frac{1}{2}\right)\left[\Delta m^{*}(n)\right]=(7.46) \mathrm{MeV}
$$

Like the proton, in the neutron being explicit the elasticity parameter $\kappa_{n}$, it is, see Equation (24):

$$
\Delta m(n)=3 \kappa_{n}\left[\Delta m^{* *}(n)\right]_{(d, \underline{d})}=3\left(\frac{7}{8}\right)(7.46) \mathrm{MeV}=(19.58) \mathrm{MeV}
$$

Thus, it is:

$m_{\text {tot }}(n)=m(n)-\Delta m_{\gamma}(n)=\{(959.23)-(19.58)\} \mathrm{MeV}=[(939.65)] \mathrm{MeV} / c^{2}$

Next to that experimental $\mathrm{m}(\mathrm{n})=(939.57) \mathrm{MeV}$.

\section{Conclusions}

In this paper, some aspects introduce a new paradigm in the phenomenology of the interactions. Describing the particles as a set of coupling quantum oscillators allows us of understanding more in-depth the Standard Model (SM) and its descriptions about the interactions, decay, spin and dynamics of particles. Besides, the AGM adds the possibilities of calculating [8] [9] the masses of hadronic particles without turning to the QCD. Not only but AGM extends the same QM, because it adds original quantum aspects as:

- The interpenetration between quarks;

- The connection between interpenetration and spin;

- The interpenetration of a quark with itself;

- The not-locality of a quark in time or the time as a quantum operator.

AGM allows explaining some aspects of the phenomenology of hadronic interactions which in contemporary literature do not have a conclusive understanding yet. For instance:

1) The spin question of the proton (mesons) has a clarifying explanation in 
Equations (3) and (21).

2) The moment questions of protons (parton model) [21], where the fraction of moment carried by the quarks is: $\left(P_{\text {quark }} / P_{p}\right) \approx(1 / 2)$. This result is a consequence of the presence of gluons in the proton. The cross-sections confirm the fact that the gluons bring a missing moment in hadron collisions. AGM explains this result, considering that along the side $\mathrm{BC}$ in Figure 3, the quantum oscillators of the junction of vertices $\mathrm{B}$ and $\mathrm{C}$ are just gluons. The side $\mathrm{BC}$ is along the propagation axis of proton-structure; thus, the moment along this axis $\left(P_{p}\right)$ is composed by the moment of gluons $\left(P_{g}\right)$ of the junction and $\left(P_{q}\right)$ moment of quarks $(u, d, u)$.

3) Some researchers, see ref. [5], have shown, with a calculation based on QCD, that quarks and gluons determine four separate contributions to the proton's mass: (a) the quark condensate ( 9\%), (b) the quark energy $(\sim 32 \%),(\mathrm{c})$ the gluonic field strength energy $(\sim 37 \%)$, and (d) the anomalous gluonic contribution ( 23\%). In AGM, the orbital rotations around the diagonal axis of quarks with their gluons of binding and junction are in accordance to this partition of mass energy (with (b), (c) and (d)). The strange quark condensate (9\%) could be, instead, according to the interpenetration of quarks with themselves (or auto-interpenetration); that recalls the self-force of classic electrodynamics.

4) The problematic phenomenology of the hadron jets [22] that reveal the close connection between quarks and gluons. In collisions at high energies the existence of gluons is in the presence of 3 coplanar hadronic jets in the system of the center of mass of the annihilation

$$
\left(e^{+} e^{-}\right): e^{+}+e^{-} \rightarrow q+q+g \rightarrow X_{\text {hadrons }}
$$

Recall both Quark and gluons, are never visible in the detector because they can only exist confined within the hadrons. In the theory of AGM is possible to interpret the jets and their hadronization as consequence of presence of lattice $\{d, \underline{d}\} \cup\{u, \underline{u}\}$. In fact, we write the reaction $\left(^{*}\right)$ as:

$$
\left(e^{+}+e^{-}\right) \rightarrow[(\gamma+\gamma) \otimes\{d, \underline{d}\} \otimes\{u, \underline{u}\}] \rightarrow q+q+g \rightarrow X_{\text {hadrons }}
$$

while, in annihilation process with proton-antiproton:

$$
\left(p^{+} \otimes p^{-}\right) \otimes[\{d, \underline{d}\} \otimes\{u, \underline{u}\}] \rightarrow\left[N^{+}+N^{-}\right]_{\{d, \underline{d}\}}+\left[X_{\text {hadrons }}\right]_{\{d, \underline{d}\}}
$$

Which we show in Figure 6.

By structure equations and geometric aspect of hadron, it is possible to find the decay probabilities.

The interpenetration of a quark with itself $\Delta m\left(q_{i} \otimes q_{i}\right)_{R}$ implies to introduce the not-local time. This, because a quark $q$ is in all times allowing to proper rotation also as it happens to spin, where the quark $q$ is in all orientations allowing to the proper rotation. This aspect is confirmed by mass defect in $\Delta m\left(q_{i} \otimes q_{i}\right)_{R}$ so as the spin, confirmed in all interactions between particles. In this way, one needs to admit the existence of time operator, see the Heinseberg relations in time version: $\Delta E \Delta t \geq 2 \pi \hbar$ The same definition of a virtual particle is so correlated to 


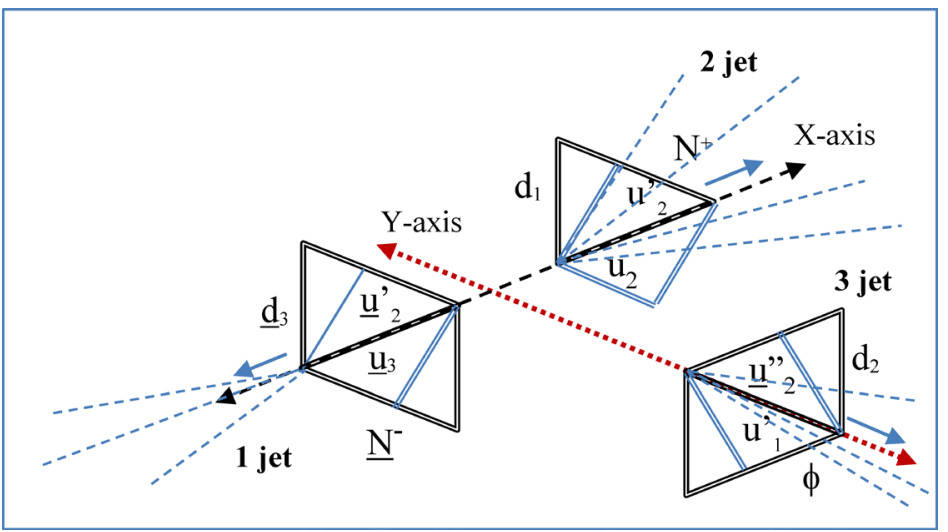

Figure 6. Production of three jets.

not-local time and, thus, time operator or the time as an observable.

Further, AGM allows explaining some problematic aspects in the hadron production accompanied by the production of the $W, Z$ bosons:

$$
p p \rightarrow q q^{-}+X+Z \rightarrow I^{+} l^{-}+X
$$

The appearance of vector bosons in hadronic jets or the transformations in beta decay induce us to introduce a hypothesis of structure in the boson particles. For example, one of the issues not yet fully clarified is the transformation of leptonic particles into quarks as happens in the production of hadronic jets:

$$
\left(e^{-}+e^{+}\right) \rightarrow \gamma+\gamma \rightarrow q+q \rightarrow \text { hadrons }
$$

How is it possible for photons to create quarks? Recall that photons are the intermediaries of the EM force. Then, we ask us because they can produce particles like quarks, provided with an "additional" agent charge, which is the color charge.

A comprehensive answer could then be to assign a structure also to a "real" elementary particle (that is not composed of sub-particles) and that it is possible to transform a structure into another only if the particles are geometric structures. Thus, there would be a mechanism of topological transformations on geometrical structures that would thus be transformed one into each other. In this way, we introduce a new paradigm in the phenomenology of strong interactions that open new perspectives for resolving the various problems of these interactions.

Note speaking of structures of quantum oscillators into particles implies (see Figure 5) a structure of quantum oscillator. On this basis, we can discuss "substructure" of quarks [19] [20] with the physical awareness that this sub-structure is realizable only through "particular" quantum oscillators. The vertex-oscillators for connecting to the other vertices through junction oscillators have to be able to have in turn a structure of "hooks": this induces us to talk about a "sub-structure" into quantum oscillator which is highlighted only into quantum oscillator coupled to other oscillators. Indication of a "composite structure" in quantum oscillator derives from its wave function. Therefore, we can describe the quantum oscillator 
with two sub-units of oscillation or "sub-oscillators". Not only but more components in an oscillator encourages us to believe that the energy of the "quanta" should be distributed between these oscillating components. The presence of more components in an oscillator causes the splitting of its quanta of energy into two, and more, sub-oscillators: this introduces the idea of half-quanta ("semiquanta") or individually half-quantum ("semi-quantum"). A quantum oscillator with a sub-structure constituted by sub-unit of oscillation, or "sub-oscillators", and "semi-quanta" is an oscillator of type "IQuO" [19] [20] [7]. To treat the particles as IQuO structures can explain the origin of some fundamental physics greatness as the electric charge, spin, isospin and color charge [23]. So, the IQuO idea constitutes a new paradigm in physics which allow us to describe with depth the physical phenomenon of particles and to open new descriptive scenarios of interactions between particles.

\section{Conflicts of Interest}

The authors declare no conflicts of interest regarding the publication of this paper.

\section{References}

[1] Kronfeld, A.S. and Quigg, C. (2010) Resource Letter QCD-1: Quantum Chromodynamics. American Journal of Physics, 78, 1081. https://doi.org/10.1119/1.3454865

[2] Udem, T. (2018) Quantum Electrodynamics and the Proton Size. Nature Physics, 14, 632. https://doi.org/10.1038/s41567-018-0166-0

[3] Tanabashi, M., et al. (2018) Review of Particle Physics. Physical Review D, 98, 030001.

[4] Walker-Loud, A. (2018) Dissecting the Mass of Proton. APS Physics, 11, 118. https://physics.aps.org/articles/v11/118

[5] Yang, Y.-B., Liang, J., Bi, Y.-J., Chen, Y., Draper, T., Liu, K.-F. and Liu, Z. (2018) Proton Mass Decomposition from the QCD Energy Momentum Tensor. Physical Review Letters, 121, Article ID: 212001. https://doi.org/10.1103/PhysRevLett.121.212001

[6] Biddle, J., Charvetto, J., Kamleh, W., Leinweber, D., et al. (2019) Publicising Lattice Field Theory through Visualisation. arXiv:1903.08308v1. https://arxiv.org/pdf/1903.08308.pdf

[7] Guido, G. (2019) The Bare and Dressed Masses of Quarks in Pions via the of Quarks' Geometric Model. Journal of High Energy Physics, Gravitation and Cosmology, 5, 1123-1149. https://doi.org/10.4236/jhepgc.2019.54065

[8] Guido, G. (2020) The Theoretical Value of Mass of the Light $\eta$-Meson via the of Quarks' Geometric Model. Journal of High Energy Physics, Gravitation and Cosmology, 6, 368-387. https://doi.org/10.4236/jhepgc.2020.63030

[9] Guido, G. (2020) The Theoretical Spectrum of Mass of the Light Mesons without Strangeness via the Quarks' Geometric Model. Journal of High Energy Physics, Gravitation and Cosmology, 6, 388-415. https://doi.org/10.4236/jhepgc.2020.63031

[10] Mohler, D. (2017) Recent Results on the Meson and Baryon Spectrum from Lattice QCD. EPJ Web of Conferences, 137, 05018.

https://doi.org/10.1051/epjconf/201713705018 
[11] Dürr, S., Fodor, Z., Frison, J., Hoelbling, C., Hoffmann, R., Katz, S.D., et al. (2008) Ab Initio Determination of Light Hadron Masses. Science, 322, 1224-1227. https://doi.org/10.1126/science.1163233

[12] Guido, G. (2017) Regarding the Structure of Quarks and Hadrons. Hadronic Journal, 40, 187-219.

[13] Guido, G. (2017) About Structure of Quarks. Hadronic Journal, 40, 221-253.

[14] Bazavov, A., Toussaint, D., Bernard, C., Laiho, J., DeTar, C., Levkova, L., et al., (2010) Nonperturbative QCD Simulations with 2+1 Flavors of Improved Staggered Quarks. Reviews of Modern Physics, 82, 1349. https://doi.org/10.1103/RevModPhys.82.1349

[15] Bazavov, A., Bernard, C., Brambilla, N., Brown, N., DeTar, C., El-Khadra, A.X., et al. (2018) Up-, Down-, Strange-, Charm-, and Bottom-Quark Masses from Four-Flavor Lattice QCD. Physical Review D, 98, 054517. https://doi.org/10.1103/PhysRevD.98.054517

[16] Ishikawa, T., Aoki, S., Fukugita, M., Hashimoto, S., Ishikawa, K.-I., Ishizuka, N., et al. (2008) Light Quark Masses from Unquenched Lattice QCD. Physical Review D, 78, 011502. https://doi.org/10.1103/PhysRevD.78.011502

[17] Kuhn, S.E., Chen, J.-P. and Leader, E. (2009) Spin Structure of the Nucleon-Status and Recent Results. Progress in Particle and Nuclear Physics, 63, 1-50. https://doi.org/10.1016/j.ppnp.2009.02.001

[18] Bradamante, F. (2008) COMPASS and HERMES Contributions to Our Understanding of the Nucleon Spin. Progress in Particle and Nuclear Physics, 61, 229-237. https://doi.org/10.1016/j.ppnp.2007.12.046

[19] Guido, G. (2012) The Substructure of a Quantum Oscillator Field. https://arxiv.org/pdf/1208.0948

[20] Guido, G. (2014) The Substructure of a Quantum Oscillator Field. Hadronic Journal, 37, 83 .

[21] Dokshitzer, Y.L., Khoze, V.A. and Troyan, S.I. (1991) On the Concept of Local Parton-Hadron Duality. Journal of Physics G: Nuclear and Particle Physics, 17, 1585. https://doi.org/10.1088/0954-3899/17/10/017

[22] Abazov, V.M., Abbott, B., Abolins, M., Acharya, B.S., Adams, M., Adams, T., et al. (2008) Measurement of the Inclusive Jet Cross-Section in pp Collisions at $(\mathrm{s})^{1 / 2}=$ 1.96 TeV. Physical Review Letters, 101, Article ID: 062001. arXiv:0802.2400v3[hep-ex]

[23] Guido, G. (2019) The Origin of the Color Charge into Quarks. Journal of High Energy Physics, Gravitation and Cosmology, 5, 1-34. https://doi.org/10.4236/jhepgc.2019.51001 